1

2

3

4

5

6

7

8

9

10

11

12

13

14

15

16

17 Word Count: 5947

18

19

$20 \quad \underline{0000-0002-2642-1351 .}$.

22

23

10

1

2

3

4

5

6

21 Correspondence concerning this article should be addressed to Jennifer T. Grant

Gambling with God: The Effect of Gambling on Religious and Spiritual Struggles Jennifer T. Grant Weinandy and Joshua B. Grubbs

Bowling Green State University (Department of Psychology), Bowling Green (Ohio), USA

$6^{\text {th }}$ January 2021

2 Weinandy, Department of Psychology, Bowling Green State University, Bowling Green, OH

43403. Contact: jtgrant@bgsu.edu 
Abstract

25 Religion and spirituality are often related to various addictive behaviors, such as substance use

26 disorders, excessive internet pornography use, and Gambling Disorder. However, presently, very

27 few published articles have considered the relationship between Gambling Disorder and negative 28 aspects of religion and spirituality such as religious and spiritual struggles. This study aimed to 29 better understand how problem gambling severity may be uniquely associated with religious and 30 spiritual struggles, both cross-sectionally and over time. The study used secondary data from a 31 longitudinal MTurk survey ( $n=764$; follow-up $n=342)$ and controlled for neuroticism, age, 32 gender, income, and gambling preference. Problem gambling severity was uniquely associated 33 with several types of religious and spiritual struggles at baseline and most struggles at a six34 month follow-up, even after controlling for baseline levels of such struggles. Further research is 35 necessary to understand the nature of these links and how they might inform clinical care. Keywords: religion, spirituality, problem gambling, religious and spiritual struggles,

\section{Gambling Disorder.}


Gambling with God: Gambling and Religious and Spiritual Struggles

Gambling Disorder (GD) is a widely recognized behavioral addiction which negatively

41 affects various aspects of well-being and predicts a range of comorbidities (see Lorains et al.,

42 2011). Therefore, understanding how GD interacts with other facets of well-being requires sustained research interest. Religious/spiritual functioning is an important domain of life

44 experience for a majority of humans (Pew Research Center, 2014). Yet, to date, there is little 45 research examining how problem gambling might influence religious well-being. The present work investigates this by examining GDs impact on religious/spiritual functioning both

47 immediately and longitudinally.

\section{Gambling within Religious and Spiritual Traditions}

Most religious texts and communities engage with the problems and effects of substance and gambling addictions both explicitly (Proverbs 20:1) and implicitly. The modern Catholic

51 tradition suggests gambling is not inherently wrong, but harming others via gambling or

52 becoming addicted is morally unacceptable (Catholic Catechism: 2413). The United Methodist

53 Church's Social Principles strongly condemns gambling while reminding followers to help those 54 with addictions (United Methodist Church, 2013). Islamic teaching views gambling as a work of

55 Satan and a sin punishable on Judgment Day (Sura Ma'idah 5:90-91). In the Jewish tradition, 56 gambling done professionally, compulsively, or for personal gain is condemned (Mishnah

57 Sanhedrin 3:3). Hindu scriptures warn against gambling and the negative consequences which may arise, both for the gambler and those around them (Rig Veda Mandala 10, Sukta 34).

59 Buddhist teachings found in the Sigalovada Sutta also speak of the dangers surrounding 60 gambling. 
63 lower levels of compulsive gambling behaviors (e.g. Braun et al., 2016; Casey et al., 2011;

64 Mutti-Packer et al., 2017) and that religious denomination may be protective against developing

65 problems with gambling (Braun et al., 2016; Eitle, 2011; Krause et al., 2017). Further,

66 religion/spirituality may be key aspects of the recovery process from gambling related problems,

67 as seen in research with those in Gambler's Anonymous (GA; e.g. Walsh, 2001), a 12-step

68 recovery organization that centers an individual's spirituality as a key element of recovery.

Despite the above findings indicating that religion/spirituality may be a buffer against

70

71

72

73

74

75

76

77

78

79

80

81

82

83

84

85

86 gambling problems, more recent research suggests the links between gambling and religion are

more nuanced. Higher religiousness may be linked to greater gambling related cognitive

distortions in some samples (Kim et al., 2018), suggesting that there are likely complex

relationships between gambling behaviors, GD, and religious/spiritual functioning. One potential relationship that has been relatively ignored in prior literature is the influence of gambling on religious and spiritual struggles.

\section{Religious and Spiritual Struggles}

Religion/spirituality can present unique challenges such as religious and spiritual struggles (RSS; e.g., Exline et al., 2014). Prior work (Exline et al., 2014) has noted that divine struggles refer to negative emotions about or toward a deity; demonic struggles focus feelings of concern about attacks from evil spirits; interpersonal struggles are focused on feelings of conflict and antipathy toward religious adherents; moral struggles refer to feelings of struggle around right and wrong; doubt struggles are feelings of distress about religious doubts; and ultimate meaning struggles are difficulties with feelings of purposelessness or lack of meaning.

Importantly, RSS are predictors of a variety of problems, such as anxiety and depression (Stauner et al., 2016; Wilt et al., 2018), higher mortality rates in chronically-ill patients (Pargament et al., 2004), poorer recovery from illness (Fitchett et al., 1999), more psychological 
87 and mental health problems (Harris et al., 2012), and neuroticism (e.g. Grubbs et al., 2016; Wilt

88 et al., 2017). These results suggest that RSS are indicators of lower psychological and physical well-being overall, and that they may drive distress and decreased psychological health (for a review see: Exline, 2013).

\section{Religious and Spiritual Struggles and Addiction}

Past work suggests that RSS are associated with addiction (e.g. Johnson et al., 2008; Krause et al., 2017), especially substance use disorders. For example, negative religious coping is linked to difficulties maintaining abstinence (Medlock et al., 2017) and can be a barrier to treatment (Puffer et al., 2012). Additionally, relationships between RSS and problem drinking seem to be stronger than the relationships between problem drinking and religious practices (Krause et al., 2017). Similarly, RSS are also linked with compulsive sexual behaviors (Griffin et al., 2016; Hook et al., 2015) and problematic pornography use (Grubbs et al., 2017), with the latter relationships being evident longitudinally as well. Collectively, these findings suggest addictions — both substance related and behavioral—are associated with greater concurrent and longitudinal experience of RSS.

Despite the above findings, relatively little work has focused on the intersection of RSS and GD. One prior study found preliminary associations between RSS and gambling addiction (Faigin et al., 2014). However, this work was a cross-sectional study of undergraduates only. Another more recent work (Gutierrez et al., 2020) demonstrated that there were relationships between higher RSS and pathological gambling in a clinical sample of U.S. veterans. Specifically, this research noted strong associations between gambling related problems and interpersonal, moral, ultimate meaning, and doubt struggles. However, these findings were limited to a very unique population (U.S. veterans receiving inpatient treatment for diagnosed GD). 


\section{The Current Study}

Building on prior research, this study aims to understand the relationship between the severity of problematic gambling and RSS in a more general sample of U.S. adults and to test these relationships over time. Previous cross-sectional research has shown that gambling is related to RSS (Faigin et al., 2014; Gutierrez et al., 2020). Building on this, we expected that problem gambling severity would be related to RSS and this relationship would continue 6 months later. That is, we were interested in examining whether or not baseline levels of problem gambling were uniquely related to RSS over time, even when baseline levels of RSS were held constant. Rather than focusing on change in RSS, our primary aim was to simply determine whether or not problem gambling was related to RSS over time. Furthermore, in designing the present work, we noted that, as previously reviewed, neuroticism is a known correlate of both gambling related problems and self-reported difficulties in religious/spiritual life (i.e. RSS;

Grubbs et al., 2016; Potenza et al., 2006; Wilt et al., 2017). Therefore, we controlled for neuroticism in all analyses.

\section{Method}

\section{Participants and Procedure}

This work made use of a pre-existing dataset, collected as a part of a larger project related to GD and Post-traumatic Stress Disorder (for full details see: https://osf.io/n29xw/). Participants from the U.S. who self-reported gambling in the past year ( $N=881$ adults) were recruited using Amazon's Mechanical Turk (MTurk) online labor marketplace through the TurkPrime data acquisition platform (Litman et al., 2017). Respondents were compensated $\$ 7.00$ for their participation. Only those who persevered through the entire study, completing the South Oaks Gambling Screen (SOGS), the RSS scale, and the baseline demographic measures (gender, age, annual income, neuroticism, and participation in online, chance, or skill-based games) were 
135 included, leaving a final sample of 764 participants (final inclusion rate=86.7\%). Six months

136 after the initial survey, participants were invited to complete a follow-up study. All 764

137 participants were contacted, of which 342 completed all follow-up measures (retention

138 rate $=44.8 \%$ ). After completing these follow-up measures, participants were compensated $\$ 5.00$

139 via the MTurk marketplace. Demographics of participants from both time points can be found in 140 Table 1.

This work was exempt from review by the authors' Institutional Review Board because it

142 solely utilized secondary data. Of note, portions of this data have been examined in prior

143 publications (Grubbs et al., 2018; Grubbs, Chapman, et al., 2019; Grubbs \& Chapman, 2019;

144 Grubbs \& Rosansky, 2019). However, no prior works based on this data have examined the role

145 of gambling in the prediction of religious/spiritual functioning.

Multivariate Analysis of Variance (MANOVA) revealed no differences on baseline

147 measures of key variables (RSS and problem gambling severity) between those who completed

148 the follow-up and those that did not (Wilk's $\lambda=0.964, F[13,329]=0.949, p=.502)$. Therefore, all

149 those who completed the baseline measures were included in the baseline analyses, regardless of

150 whether they completed the follow-up measures.

151 Measures

Table 2 shows means, standard deviations, ranges, and internal consistency values for 153 neuroticism, SOGS and each RSS subscale at baseline and follow-up.

Problem Gambling Severity. Problem gambling severity was assessed using the South

155 Oaks Gambling Screen (SOGS; Lesieur \& Blume, 1987). The SOGS contains 21-items, of which

156 only 20 are scored, asking participants questions such as "Have people criticized your

157 gambling?" and "Did you ever gamble more than you intended to?". Responses consistent with 
GD were given a value of 1 . Responses were summed and ranged from 0 to $20{ }^{1}$

Religious and Spiritual Struggles. The 26-item RSS scale (Exline et al., 2014) includes

six subscales (Divine: 5 items, Demonic: 4 items, Interpersonal: 5 items, Moral: 4 items,

Ultimate Meaning: 4 items, and Doubt: 4 items) which were analyzed separately for this study, similar to other studies looking at addiction and RSS (e.g. Stauner et al., 2019). Participants rated each statement from 1 (not at all) to 5 (a great deal), and mean scores were calculated for each of the subscales.

Neuroticism. The International Personality Inventory Pool NEO 120 (IPIP-NEO-120; Maples, Guan, Carter, \& Miller, 2014) was included as a measure of neuroticism. The six facet scores of the Neuroticism index (Anxiety, Anger, Depression, Self-consciousness, Immoderation, and Vulnerability) were averaged to create a composite Neuroticism scale score. Type of Gambling. Participants were asked to indicate their preferred method and type of gambling. Participants were coded as gambling online if they responded that they had most often gambled online in the past 12 months, all others were coded as not primarily gambling online. Participants were additionally coded as either primarily preferring chance games (i.e., the lottery, bingo, dice, slots, tabs, keno, scratch-offs), skill-based games (i.e., played cards, bet on horse/dog races, sports betting, prop betting, stocks, fantasy sports), or both chance-based and skill-based games equally (equal preference).

\section{Plan for Analyses}

For all variables, we first computed descriptive statistics and Pearson correlations. To test key relationships, we followed the same analytic strategy employed in past work on behavioral addiction and RSS (e.g. Grubbs et al., 2017). We first conducted a series of cross-sectional

\footnotetext{
${ }^{1}$ Authors also analysed the data after excluding all individuals who reported no problem gambling (SOGS $\left.<1\right)$, leaving a sample size of 602. These results (available at: https://osf.io/srvd7/) were very similar to the results presented here, thus we have presented those with the highest power.
} 
hierarchical regressions between key predictors at Time 1 and each RSS at Time 1 . In the first step of the regressions, we included neuroticism, gender, age, annual income, and preference for online, chance, or skill-based games as control variables. Baseline levels of problem gambling severity (using the SOGS) was entered in the second step.

Again consistent with prior work (Grubbs et al., 2017), we then sought to examine the unique role of gambling problems in predicting each RSS score at Time 2, while holding the same RSS scores at Time 1 constant, using hierarchical regressions. Given that only two timepoints were available, that the variables may not be stable over time, and that our aim was to determine the unique role of gambling problems in predicting RSS over time, rather than evaluating the effect of an intervention or change, such a residualized change approach is adequate (Castro-Schilo \& Grimm, 2018; Gollwitzer et al., 2014). In the first step of these regressions, we included the control variables and baseline levels of the RSS being predicted. ${ }^{2}$ In the subsequent step, baseline levels of problem gambling severity were entered.

\section{Results}

Although this study utilized the whole sample and aimed to use any level of reported gambling problems, most participants did not report experiencing clinically significant levels of problem gambling. Based on previously established cut-off points for clinically significant gambling problems on the SOGS (Goodie et al., 2013), 27\% scored above a liberal cut-off of 5, $15 \%$ scored above a cut-off of 8 , and $6 \%$ scored above a cut-off of 12 , which has been suggested for MTurk samples. These percentages are slightly above rates found in the general population (e.g. 2.7\%-5.6\%: Welte et al., 2015).

\footnotetext{
${ }^{2}$ Authors also analysed the RSS at Time 2 after including all RSS at baseline in step 1 (Supplementary Table 5: https://osf.io/srvd7/), results of which were similar to those presented here.
} 
GAMBLING WITH GOD

\section{Correlational Analyses}

Table 2 shows correlations between baseline levels of neuroticism and problem gambling severity, and all six RSS at both time points.

Analyses revealed small positive correlations between problem gambling severity and neuroticism. Results showed medium-to-large positive correlations between the various RSS and between RSS at baseline and follow-up. Each RSS correlated highly and positively with later reports of the same RSS. Neuroticism was positively correlated with all RSS at both time points. Furthermore, problem gambling severity demonstrated small-to-medium positive correlations with all RSS at both time points.

\section{Hierarchical Regressions}

Baseline. Table 3 shows standard regression estimates (i.e., beta weights) and full regression results for all hierarchical regressions with baseline data.

Across all six struggles, neuroticism emerged as a significant predictor in the first step of the regression. In the subsequent step, SOGS scores emerged as a significant predictor for all struggles, accounting for $2.4 \%$ (interpersonal struggles) to $10.4 \%$ (demonic struggles) of unique variance above control variables.

Longitudinal. Table 4 shows standard regression estimates (i.e., beta weights) and full regression results for all follow-up level hierarchical regression analyses.

Across all six follow-up struggles, neuroticism (except for demonic struggles) and baseline levels of the same struggle emerged as significant predictors in the first step of the regression. In the subsequent step, SOGS scores emerged as a significant predictor for all struggles except ultimate meaning struggles, accounting for $0.6 \%$ (interpersonal struggles) to $2 \%$

(doubt struggles) of unique variance above control variables and baseline levels of the same struggle. 


\section{Discussion}

This work aimed to better understand the relationship between gambling and RSS in the

U.S., cross-sectionally and longitudinally. Problem gambling severity was significantly and

uniquely associated with all six RSS at baseline and all RSS, except ultimate meaning struggles, at the six-month follow-up. Moreover, raw correlations between problem gambling and RSS at both baseline and follow-up were highly comparable, suggesting robust and consistent associations between these domains over time.

Behavioral Addictions and Religious and Spiritual Struggles. Prior works have shown that, with regards to addiction, value inconsistent behaviors often lead to a variety of intrapersonal problems (e.g. Grubbs, Kraus, et al., 2019). For example, previous research has

236 found that perceived problems with internet pornography predicted divine, moral, and 237 interpersonal struggles cross-sectionally and moral and interpersonal struggles longitudinally 238 (Grubbs et al., 2017). The present study extends those findings to another domain of behavioral dysregulation: problem gambling. Moreover, this study found links between problem gambling and other RSS over time (i.e., not just moral and interpersonal struggles).

Previously identified correlations specifically between gambling and divine RSS (Faigin

242 et al., 2014; Gutierrez et al., 2020) were replicated here. This study extends these prior works

243 finding that most of these relationships continued at a six-month follow-up. This suggests the 244 relationship between gambling and RSS persists over time and may provide preliminary 245 evidence for potential causal pathways. Although a two-time-point analysis does not allow us to 246 draw causal inferences, the longitudinal nature of the findings suggest a robust relationship

247 between these two domains. Additionally, the online sample of adults used in this study increases 248 generalizability of these results over prior works which were limited to either undergraduates 249 (Faigin et al., 2014) or treatment seeking U.S. Armed Forces veterans (Gutierrez et al., 2020). In 
sum, the findings of the present work, taken alongside the cross-sectional, longitudinal, and clinical samples discussed above, suggests there are important links between self-reported addictive behaviors and the experience of RSS.

\section{Implications}

Previous research suggests that problem gambling can cause distress in multiple areas of life, including increasing criminal and suicidal behaviors, general psychological distress, and decreasing overall well-being (Battersby et al., 2006; Black et al., 2013; Kessler et al., 2008; Laursen et al., 2016). Therefore, treatment tends to focus on the gambler's relationships, mental health, and financial well-being. Unfortunately, the published literature suggests that the domain of religion/spirituality has been largely ignored in the clinical treatment of GD. However, the findings of this study and prior studies (i.e., Gutierrez et al., 2020) suggest that RSS may be salient for clinicians' treatment of problematic gambling. This is especially clinically relevant given that RSS often predict other psychological problems. More broadly, this work supports, as many prior works have also suggested, the need for spiritually integrated care in mental health treatment settings that allows for the assessment, acknowledgment, and exploration of clients' religious/spiritual beliefs (e.g. Pargament, 2007). Furthermore, GA, which already has some ties to addressing religion/spirituality, may be particularly poised to address some of these concerns.

\section{Limitations and Constraints on Generalizability}

This study used self-report measures, which have well-known limitations (Chan, 2009). Potential problems with MTurk data, including data quality issues and concerns about representativeness, have been noted in previous work; however, validity checks, which were used in this data collection, may somewhat mitigate these (e.g. Chmielewski \& Kucker, 2020).

Since this sample was from the U.S., caution should be taken when generalizing these results to other countries. 
This study included only two time points, precluding growth curve modeling or causal

275 inferences. Admittedly, the use of hierarchical regressions to predict future RSS through residualized change is only one of many possible approaches to longitudinal data. Latent change

277 scores, difference score models, and simple pre-and-post comparisons are each potential ways to 278 analyze this same data (Castro-Schilo \& Grimm, 2018; Gollwitzer et al., 2014). However, given 279 that our aim was to determine whether gambling related problems predicted unique variance in RSS over time, using simple hierarchical regressions was the most parsimonious approach.

Religiousness, including religious attendance and strength of belief, was not included as a control variable in the present study, as it was not included in this dataset. This is a particular concern because prior works have shown that religiousness has a significant effect on an

284 individual's participation in gambling and their experiences of RSS (Exline et al., 2014; Lam, 2006). However, prior work has noted that RSS are not just symptoms of distress in religious populations (Stauner et al., 2016). Rather, RSS are unique phenomena, that occur more often in religious populations, represent distinct distress, and predict salient mental health outcomes, even when controlling for religiousness (Exline et al., 2014). Future studies should be aware that this would be a useful control variable and may have accounted for a significant amount of the unexplained variance in this study.

The mean values of the RSS scale and SOGS were low (below the midpoint of the scale).

292 As such, these results should be viewed with caution as they may not be representative of 293 individuals who score highly on either scale. This may also explain the discrepancy between 294 these results and those of Gutierrez et al. (2020).

The amount of variance in struggles accounted for by problem gambling severity in the regressions was small-to-moderate (i.e., $2.4 \%$ to $10.4 \%$ at baseline; $0.6 \%$ to $2 \%$ over time). As 297 such, although gambling behavior is likely related to RSS, further research is necessary to fully 
GAMBLING WITH GOD

298 understand the practical effect of this relationship. Even so, the longitudinal regression analyses

299 were conservative tests, as they controlled for baseline levels of RSS, and therefore small effect

300 sizes should be expected.

301 Conclusion

302

Both gambling and RSS have been shown to predict significant psychological distress,

303 such as depression and anxiety (e.g. Kessler et al., 2008). Historically, many religious/spiritual

304 groups have viewed gambling in a negative light and shunned those who participated. As such,

305 those who gamble may find themselves at odds with their religion/spirituality, leading to further

306 distress. This study looked at how much problem gambling severity was uniquely associated

307 with RSS using correlational and hierarchical regression analyses. The results showed that

308 gambling problem severity was uniquely associated with RSS cross-sectionally and continued,

309 except for ultimate meaning struggles, after six months. Overall, researchers should continue to

310 investigate how to reduce RSS, particularly in those with GD, and examine how religiousness

311 may affect this relationship. Results from this study suggest that, despite being largely ignored to

312 date, the domain of religion/spirituality, and in particular RSS, should be given attention when

313 considering problematic gambling behaviors. 
Declarations

$316 \quad$ Funding

317 We thank the International Center for Responsible Gaming (ICRG) for the seed grant

318 awarded to Joshua B. Grubbs that funded the collection of this dataset.

319 Conflicts of Interest

320 The authors declare that they have no conflicts of interest.

321 Ethics Approval

322 Ethical approval was waived by the Institutional Review Board at Bowling Green State

323 University for this work because it solely utilized secondary data and, therefore, did not meet the 324 definition for human subjects' research.

325 Availability of data and material

326 Data will be made available upon request to the corresponding author.

327 Authors Contributions

328 JTGW: Conceptualization, methodology, formal analysis, data curation, writing,

329 visualization, project administration; JBG: Conceptualization, validation, investigation,

330 resources, data acquisition, writing-review and editing, supervision, funding acquisition.

331 Acknowledgements

332 We are grateful for Harold Rosenberg's and Dara Musher-Eizenman's helpful comments

333 on an earlier version of this manuscript. 


\section{References}

Battersby, M., Tolchard, B., Scurrah, M., \& Thomas, L. (2006). Suicide ideation and behaviour in people with pathological gambling attending a treatment service. International Journal of Mental Health and Addiction, 4(3), 233-246. https://doi.org/10.1007/s11469-006-9022-z

Black, D. W., Shaw, M., McCormick, B., \& Allen, J. (2013). Pathological gambling:

Relationship to obesity, self-reported chronic medical conditions, poor lifestyle choices, and impaired quality of life. Comprehensive Psychiatry, 54(2), 97-104. https://doi.org/10.1016/j.comppsych.2012.07.001

Braun, B., Kornhuber, J., \& Lenz, B. (2016). Gaming and religion: The impact of spirituality and denomination. Journal of Religion and Health, 55(4), 1464-1471. https://doi.org/10.1007/s10943-015-0152-0

Casey, D. M., Williams, R. J., Mossière, A. M., Schopflocher, D. P., El-Guebaly, N., Hodgins, D. C., Smith, G. J., \& Wood, R. T. (2011). The role of family, religiosity, and behavior in adolescent gambling. Journal of Adolescence, 34(5), 841-851. https://doi.org/10.1016/j.adolescence.2011.02.002

Castro-Schilo, L., \& Grimm, K. J. (2018). Using residualized change versus difference scores for longitudinal research. Journal of Social and Personal Relationships, 35(1), 32-58. https://doi.org/10.1177/0265407517718387

Chan, D. (2009). So why ask me? Are self-report data really that bad? In Statistical and methodological myths and urban legends: Doctrine, verity and fable in the organizational and social sciences (pp. 309-336). Routledge/Taylor \& Francis Group.

Chmielewski, M., \& Kucker, S. C. (2020). An MTurk crisis? Shifts in data quality and the impact on study results. Social Psychological and Personality Science, 11(4), 464-473. https://doi.org/10.1177/1948550619875149 
Eitle, D. (2011). Religion and gambling among young adults in the United States: Moral communities and the deterrence hypothesis. Journal for the Scientific Study of Religion, 50(1), 61-81. https://doi.org/10.1111/j.1468-5906.2010.01552.x

Exline, J. J. (2013). Religious and spiritual struggles. In J. J. Exline (Ed.), APA handbook of psychology, religion, and spirituality (Vol 1): Context, theory, and research. (Vol. 1, pp. 459-475). American Psychological Association. https://doi.org/10.1037/14045-025

Exline, J. J., Pargament, K. I., Grubbs, J. B., \& Yali, A. M. (2014). The religious and spiritual struggles scale: Development and initial validation. Psychology of Religion and Spirituality, 6(3), 208-222. https://doi.org/10.1037/a0036465

Faigin, C. A., Pargament, K. I., \& Abu-Raiya, H. (2014). Spiritual struggles as a possible risk factor for addictive behaviors: An initial empirical investigation. The International Journal for the Psychology of Religion, 24(3), 201-214. https://doi.org/10.1080/10508619.2013.837661

Fitchett, G., Rybarczyk, B. D., DeMarco, G. A., \& Nicholas, J. J. (1999). The role of religion in medical rehabilitation outcomes: A longitudinal study. Rehabilitation Psychology, 44(4), 333-353. https://doi.org/10.1037/0090-5550.44.4.333

Gollwitzer, M., Christ, O., \& Lemmer, G. (2014). Individual differences make a difference: On the use and the psychometric properties of difference scores in social psychology. European Journal of Social Psychology, 44(Methods and statistics in social psychology: Refinements and new developments), 673-482.

Goodie, A. S., MacKillop, J., Miller, J. D., Fortune, E. E., Maples, J. L., Lance, C. E., \& Campbell, W. K. (2013). Evaluating the south oaks gambling screen with DSM-IV and DSM-5 criteria: Results from a diverse community sample of gamblers. Assessment, 20(5), 523-531. https://doi.org/10.1177/1073191113500522 
Griffin, B. J., Worthington, E. L., Leach, J. D., Hook, J. N., Grubbs, J., Exline, J. J., \& Davis, D. E. (2016). Sexual congruence moderates the associations of hypersexual behavior with spiritual struggle and sexual self-concept. Sexual Addiction and Compulsivity, 23(2-3), 279-295. https://doi.org/10.1080/10720162.2016.1150924

Grubbs, J. B., \& Chapman, H. (2019). Predicting gambling situations: The roles of impulsivity, substance use, and post-traumatic stress. Substance Abuse: Research and Treatment, 13. https://doi.org/10.1177/1178221819852641

Grubbs, J. B., Chapman, H., Milner, L., Gutierrez, I. A., \& Bradley, D. F. (2018). Examining links between posttraumatic stress and gambling motives: The role of positive gambling expectancies. Psychology of Addictive Behaviors, 32(7), 821-831. https://doi.org/10.1037/adb0000399

Grubbs, J. B., Chapman, H., \& Shepherd, K. A. (2019). Post-traumatic stress and gambling related cognitions: Analyses in inpatient and online samples. Addictive Behaviors, 89, 128135. https://doi.org/10.1016/j.addbeh.2018.09.035

Grubbs, J. B., Exline, J. J., Pargament, K. I., Volk, F., Lindberg, M. J., Grubbs, J. B., Exline, J. J., Pargament, K. I., \& Lindberg, M. J. (2017). Internet pornography use, perceived addiction, and religious/spiritual struggles. Archives of Sexual Behavior, 46(6), 1733-1745. https://doi.org/10.1007/s10508-016-0772-9

Grubbs, J. B., Kraus, S. W., \& Perry, S. L. (2019). Self-reported addiction to pornography in a nationally representative sample: The roles of use habits, religiousness, and moral incongruence. Journal of Behavioral Addictions, 1-6. https://doi.org/10.1556/2006.7.2018.134

Grubbs, J. B., \& Rosansky, J. A. (2019). Problem gambling, coping motivations, and positive expectancies: A longitudinal survey study. Psychology of Addictive Behaviors. 
GAMBLING WITH GOD

https://doi.org/10.1037/adb0000529

Grubbs, J. B., Wilt, J., Stauner, N., Exline, J. J., \& Pargament, K. I. (2016). Self, struggle, and soul: Linking personality, self-concept, and religious/spiritual struggle. Personality and Individual Differences, 101, 144-152. https://doi.org/10.1016/j.paid.2016.05.365

Gutierrez, I. A., Chapman, H., Grubbs, J. B., \& Grant, J. (2020). Religious and spiritual struggles among military veterans in a residential gambling treatment programme. Mental Health, Religion and Culture, 1-17. https://doi.org/10.1080/13674676.2020.1764513

Harris, J. I., Erbes, C. R., Engdahl, B. E., Ogden, H., Olson, R. H. A., Winskowski, A. M. M., Campion, K., \& Mataas, S. (2012). Religious distress and coping with stressful life events: A longitudinal study. Journal of Clinical Psychology, 68(12), 1276-1286. https://doi.org/10.1002/jclp.21900

Hook, J. N., Farrell, J. E., Ramos, M. J., Davis, D. E., Karaga, S., Van Tongeren, D. R., \& Grubbs, J. (2015). Religiousness and congruence between sexual values and behavior. Journal of Psychology and Christianity, 34(2), 179-188.

Johnson, T. J., Sheets, V. L., \& Kristeller, J. L. (2008). Empirical identification of dimensions of religiousness and spirituality. Mental Health, Religion \& Culture, 11(8), 745-767. https://doi.org/10.1080/13674670701561209

Kessler, R. C., Hwang, I., Labrie, R., Petukhova, M., Sampson, N. A., Winters, K. C., \& Shaffer, H. J. (2008). DSM-IV pathological gambling in the National Comorbidity Survey Replication. Psychological Medicine, 38(9), 1351-1360. https://doi.org/10.1017/S0033291708002900

Kim, H. S., Shifrin, A., Sztainert, T., \& Wohl, M. J. A. (2018). Placing your faith on the betting floor: Religiosity predicts disordered gambling via gambling fallacies. Journal of Behavioral Addictions, 1-9. https://doi.org/10.1556/2006.7.2018.23 
GAMBLING WITH GOD

430

431

432

433

434

435

436

437

438

439

440

441

442

443

444

445

446

447

448

449

450

451

452

453

Krause, N., Pargament, K. I., Hill, P. C., \& Ironson, G. (2017). Spiritual struggles and problem drinking: Are younger adults at greater risk than older adults? Substance Use \& Misuse, 18. https://doi.org/10.1080/10826084.2017.1387567

Lam, D. (2006). The influence of religiosity on gambling participation. Journal of Gambling Studies, 22(3), 305-320. https://doi.org/10.1007/s10899-006-9018-4

Laursen, B., Plauborg, R., Ekholm, O., Larsen, C. V. L., \& Juel, K. (2016). Problem gambling associated with violent and criminal behaviour: A danish population-based survey and register study. Journal of Gambling Studies, 32(1), 25-34. https://doi.org/10.1007/s 10899015-9536-Z

Lesieur, H. R., \& Blume, S. (1987). The south oaks gambling screen (SOGS): A new instrument for the identification of pathological gamblers. American Journal of Psychiatry, 144(9), $1184-1188$.

Litman, L., Robinson, J., \& Abberbock, T. (2017). TurkPrime.com: A versatile crowdsourcing data acquisition platform for the behavioral sciences. Behavior Research Methods, 49(2), 433-442. https://doi.org/10.3758/s13428-016-0727-z

Lorains, F. K., Cowlishaw, S., \& Thomas, S. A. (2011). Prevalence of comorbid disorders in problem and pathological gambling: Systematic review and meta-analysis of population surveys. In Addiction (Vol. 106, Issue 3, pp. 490-498). https://doi.org/10.1111/j.13600443.2010.03300.x

Maples, J. L., Guan, L., Carter, N. T., \& Miller, J. D. (2014). A test of the international personality item pool representation of the revised NEO personality inventory and development of a 120-item IPIP-based measure of the five-factor model. Psychological Assessment, 26(4), 1070-1084. https://doi.org/10.1037/pas0000004

Medlock, M. M., Rosmarin, D. H., Connery, H. S., Griffin, M. L., Weiss, R. D., Karakula, S. L., 
GAMBLING WITH GOD

\& McHugh, R. K. (2017). Religious coping in patients with severe substance use disorders receiving acute inpatient detoxification. American Journal on Addictions, 26(7), 744-750. https://doi.org/10.1111/ajad.12606

Mutti-Packer, S., Hodgins, D. C., Williams, R. J., \& Thege, B. K. (2017). The protective role of religiosity against problem gambling: Findings from a five-year prospective study. $B M C$ Psychiatry, 17(1), 356. https://doi.org/10.1186/s12888-017-1518-5

Pargament, K. I. (2007). Spiritually integrated psychotherapy: Understanding and addressing the sacred. Guilford Press.

Pargament, K. I., Koenig, H. G., Tarakeshwar, N., \& Hahn, J. (2004). Religious coping methods as predictors of psychological, physical and spiritual outcomes among medically ill elderly patients: A two-year longitudinal study. Journal of Health Psychology, 9(6), 713-730. https://doi.org/10.1177/1359105304045366

Pew Research Center. (2014). Importance of religion in one's life: Religious Data, Demographics and Statistics. https://www.pewforum.org/religious-landscapestudy/importance-of-religion-in-ones-life/

Potenza, M. N., Steinberg, M. A., Wu, R., Rounsaville, B. J., \& O’Malley, S. S. (2006). Characteristics of older adult problem gamblers calling a gambling helpline. Journal of Gambling Studies, 22(2), 241-254. https://doi.org/10.1007/s10899-006-9013-9

Puffer, E. S., Skalski, L. M., \& Meade, C. S. (2012). Changes in religious coping and relapse to drug use among opioid-dependent patients following inpatient detoxification. Journal of Religion and Health, 51(4), 1226-1238. https://doi.org/10.1007/s10943-010-9418-8

Stauner, N., Exline, J., Grubbs, J., Pargament, K., Bradley, D., \& Uzdavines, A. (2016). Bifactor models of religious and spiritual struggles: Distinct from religiousness and distress. Religions, 7(6), 68. https://doi.org/10.3390/rel7060068 
GAMBLING WITH GOD

478 Stauner, N., Exline, J., Kusina, J. R., \& Pargament, K. I. (2019). Religious and spiritual

479

480

481

482

483

484

485

486

487

488

489

490

491

492

493

494

495 struggles, religiousness, and alcohol problems among undergraduates. Journal of Prevention and Intervention in the Community, 47(3), 243-258. https://doi.org/10.1080/10852352.2019.1603678

Walsh, J. M. (2001). Spirituality and recovery from pathological gambling. Loyola College in Maryland.

Welte, J. W., Barnes, G. M., Tidwell, M. C. O., Hoffman, J. H., \& Wieczorek, W. F. (2015). Gambling and problem gambling in the United States: Changes between 1999 and 2013. Journal of Gambling Studies, 31(3), 695-715. https://doi.org/10.1007/s10899-014-9471-4

Wilt, J. A., Grubbs, J. B., Pargament, K. I., \& Exline, J. J. (2017). Religious and spiritual struggles, past and present: Relations to the big five and well-being. International Journal for the Psychology of Religion, 27(1), 51-64. https://doi.org/10.1080/10508619.2016.1183251

Wilt, J. A., Stauner, N., Lindberg, M. J., Grubbs, J. B., Exline, J. J., \& Pargament, K. I. (2018). Struggle with ultimate meaning: Nuanced associations with search for meaning, presence of meaning, and mental health. Journal of Positive Psychology, 13(3), 240-251. https://doi.org/10.1080/17439760.2017.1279208 
Table 1.

Demographics of Participants at Baseline and Follow-up Time Points.

\begin{tabular}{|c|c|c|}
\hline & Baseline $^{\mathrm{a}}$ & Follow-up ${ }^{b}$ \\
\hline \multicolumn{3}{|l|}{ Gender } \\
\hline Female & $412(54 \%)$ & $185(54 \%)$ \\
\hline Male & $352(46 \%)$ & $157(46 \%)$ \\
\hline Age & $36.1(\mathrm{SD}=11.2)$ & $37.4(\mathrm{SD}=11.3)$ \\
\hline \multicolumn{3}{|l|}{ Race/Ethnicity } \\
\hline White/Caucasian & $584(76 \%)$ & $261(76 \%)$ \\
\hline African-American/Black & $89(12 \%)$ & $40(12 \%)$ \\
\hline Asian/Pacific Islander & $66(9 \%)$ & $34(10 \%)$ \\
\hline Latino/Hispanic & $63(8 \%)$ & $29(9 \%)$ \\
\hline American Indian/Native-American/Alaska Native & $12(2 \%)$ & $3(1 \%)$ \\
\hline Middle Eastern & $3(0 \%)$ & $0(0 \%)$ \\
\hline Other & $10(2 \%)$ & $3(1 \%)$ \\
\hline \multicolumn{3}{|l|}{ Sexual Orientation } \\
\hline Heterosexual & $664(87 \%)$ & $302(88 \%)$ \\
\hline Homosexual & $33(4 \%)$ & $12(4 \%)$ \\
\hline Bisexual & $56(7 \%)$ & $25(7 \%)$ \\
\hline Asexual & $3(0 \%)$ & $1(0 \%)$ \\
\hline Pansexual & $5(1 \%)$ & $1(0 \%)$ \\
\hline Other & $4(0 \%)$ & $1(0 \%)$ \\
\hline \multicolumn{3}{|l|}{ Relationship/Marital Status } \\
\hline Married & $271(36 \%)$ & $138(40 \%)$ \\
\hline Single, not in a committed relationship & $241(32 \%)$ & $103(30 \%)$ \\
\hline Single and in a committed relationship & $122(16 \%)$ & $45(13 \%)$ \\
\hline Living with a partner & $115(15 \%)$ & $46(14 \%)$ \\
\hline Divorced & $47(6 \%)$ & $29(9 \%)$ \\
\hline Separated & $9(1 \%)$ & $5(2 \%)$ \\
\hline Widowed & $6(1 \%)$ & $2(1 \%)$ \\
\hline Average Annual Income & $\begin{array}{l}\$ 56,175 \\
(\mathrm{SD}=\$ 44,039)\end{array}$ & $\begin{array}{l}\$ 59,145 \\
(\mathrm{SD}=\$ 48,935)\end{array}$ \\
\hline \multicolumn{3}{|l|}{ Preferred game type } \\
\hline Chance games & $448(59 \%)$ & $202(59 \%)$ \\
\hline Skill-based games & $168(22 \%)$ & $85(25 \%)$ \\
\hline Equal preference & $148(19 \%)$ & $55(16 \%)$ \\
\hline Online games & $59(8 \%)$ & $26(8 \%)$ \\
\hline
\end{tabular}

Note: ${ }^{\mathrm{a}} \mathrm{N}=764 ;{ }^{\mathrm{b}} \mathrm{N}=342$ 
Table 2.

Descriptive Statistics for and Correlations Between Neuroticism, SOGS and RSS Subscale Measures.

\begin{tabular}{|c|c|c|c|c|c|c|c|c|c|c|c|}
\hline & $\operatorname{Mean}(S D)$ & $\alpha$ & Observed Range & 1 & 2 & 3 & 4 & 5 & 6 & 7 & 8 \\
\hline \multicolumn{12}{|c|}{ Baseline $^{\mathrm{a}}$} \\
\hline 1. Neuroticism & $2.71(0.85)$ & .94 & $1-4.92$ & - & & & & & & & \\
\hline 2. SOGS & $3.50(4.03)$ & .90 & $0-20$ & .132 & - & & & & & & \\
\hline \multicolumn{12}{|l|}{ RSS } \\
\hline 3. Divine & $1.43(0.84)$ & .93 & $1-5$ & .277 & .319 & - & & & & & \\
\hline 4. Demonic & $1.42(0.85)$ & .94 & $1-5$ & .172 & .364 & .622 & - & & & & \\
\hline 5. Interpersonal & $1.69(0.89)$ & .87 & $1-5$ & .298 & .215 & .537 & .421 & - & & & \\
\hline 6. Moral & $1.71(0.99)$ & .92 & $1-5$ & .278 & .337 & .611 & .661 & .476 & - & & \\
\hline 7. Ultimate Meaning & $1.89(1.06)$ & .89 & $1-5$ & .520 & .244 & .573 & .386 & .546 & .527 & - & \\
\hline 8. Doubt & $1.57(0.91)$ & .92 & $1-5$ & .325 & .274 & .732 & .500 & .584 & .624 & .609 & - \\
\hline \multicolumn{12}{|c|}{ Follow-up ${ }^{b}$} \\
\hline \multicolumn{12}{|l|}{ RSS } \\
\hline 9. Divine & $1.34(0.74)$ & .95 & $1-5$ & .261 & .369 & .644 & .463 & .381 & .365 & .355 & .427 \\
\hline 10. Demonic & $1.32(0.70)$ & .94 & $1-5$ & .168 & .394 & .414 & .604 & .344 & .386 & .224 & .308 \\
\hline 11. Interpersonal & $1.50(0.74)$ & .87 & $1-4.60$ & .330 & .267 & .350 & .379 & .642 & .337 & .388 & .372 \\
\hline 12. Moral & $1.54(0.86)$ & .92 & $1-4.75$ & .268 & .375 & .350 & .460 & .309 & .610 & .327 & .428 \\
\hline 13. Ultimate Meaning & $1.76(0.98)$ & .90 & $1-5$ & .520 & .237 & .407 & .263 & .362 & .309 & .619 & .409 \\
\hline 14. Doubt & $1.43(0.73)$ & .90 & $1-4.25$ & .338 & .365 & .562 & .405 & .421 & .446 & .433 & .655 \\
\hline
\end{tabular}

Note: ${ }^{\mathrm{a}} N=764 ;{ }^{\mathrm{b}} N=342 ;$ All correlations are statistically significant $(p<.01)$ 
Table 3.

Summary of Hierarchical Regression Analysis of Variables Predicting All Six RSS at Baseline.

\begin{tabular}{|c|c|c|c|c|c|c|}
\hline \multicolumn{7}{|c|}{ STEP 1} \\
\hline Variable & $\begin{array}{l}\text { RSS } \\
\text { Divine }\end{array}$ & $\begin{array}{l}\text { RSS } \\
\text { Demonic }\end{array}$ & $\begin{array}{l}\text { RSS } \\
\text { Interpersonal }\end{array}$ & $\begin{array}{l}\text { RSS } \\
\text { Moral }\end{array}$ & $\begin{array}{l}\text { RSS } \\
\text { Meaning }\end{array}$ & $\begin{array}{l}\text { RSS } \\
\text { Doubt }\end{array}$ \\
\hline Neuroticism & $.267 * *$ & $.147 * *$ & $.298 * *$ & $.272 * *$ & $.528 * *$ & $.313 * *$ \\
\hline Gender & .028 & -.027 & .062 & .045 & $.096 * *$ & .014 \\
\hline Online Gambler & $.119 * *$ & $.121 * *$ & $.089 *$ & .066 & .030 & $.091 * *$ \\
\hline Chance Games & .010 & .046 & .019 & .020 & $-.102 *$ & .005 \\
\hline Skill Games & .055 & $.100 *$ & .002 & .076 & -.078 & .037 \\
\hline Age & .001 & -.019 & .001 & -.032 & .005 & -.006 \\
\hline Annual Income & $-.079 *$ & $-.097 * *$ & -.044 & -.068 & $-.081 * *$ & $-.077 *$ \\
\hline$R^{2}$ & .101 & .060 & .103 & .096 & .297 & .122 \\
\hline$F$ for $R^{2}$ & $12.13 * *$ & $6.83 * *$ & $12.43 * *$ & $11.45^{* *}$ & $45.54 * *$ & $14.97 * *$ \\
\hline \multicolumn{7}{|c|}{ STEP 2} \\
\hline Neuroticism & $.232 * *$ & $.103 * *$ & $.276^{* *}$ & $.233 * *$ & $.506^{* *}$ & $.284 * *$ \\
\hline Gender & .007 & -.053 & .050 & .023 & $.083 *$ & -.002 \\
\hline Online Gambler & .060 & .048 & .054 & .001 & .007 & .042 \\
\hline Chance Games & .004 & .039 & .015 & .014 & $-.105 * *$ & .000 \\
\hline Skill Games & .026 & .064 & -.015 & .044 & $-.096 . *$ & .014 \\
\hline Age & .005 & -.015 & .004 & -.028 & .008 & -.003 \\
\hline Annual Income & $-.075^{*}$ & $-.092 * *$ & -.042 & -.065 & $-.078 * *$ & $-.074 *$ \\
\hline SOGS & $.270 * *$ & $.337 * *$ & $.163 * *$ & $.297 * *$ & $.171 * *$ & $.223 * *$ \\
\hline$R^{2}$ & .168 & .163 & .128 & .176 & .323 & .167 \\
\hline$\Delta R^{2}$ & .067 & .104 & .024 & .080 & .027 & .045 \\
\hline$F$ for $\Delta R^{2}$ & $60.42 * *$ & $93.54 * *$ & $21.13 * *$ & $73.61 * *$ & $29.71 * *$ & $41.04 * *$ \\
\hline
\end{tabular}

Note: $* * p \leq 0.01 ; * p \leq 0.05 ; N=764$

Problem gambling severity in bold typeface for clarity. 
Table 4.

Summary of Hierarchical Regression Analysis of Variables Predicting All Six RSS at Followup after Controlling for Baseline Levels of the Same RSS.

\begin{tabular}{|c|c|c|c|c|c|c|}
\hline \multicolumn{7}{|c|}{ STEP 1} \\
\hline Variable & $\begin{array}{l}\text { RSS } \\
\text { Divine }\end{array}$ & $\begin{array}{l}\text { RSS } \\
\text { Demonic }\end{array}$ & $\begin{array}{l}\text { RSS } \\
\text { Interpersonal }\end{array}$ & $\begin{array}{l}\text { RSS } \\
\text { Moral }\end{array}$ & $\begin{array}{l}\text { RSS } \\
\text { Meaning }\end{array}$ & $\begin{array}{l}\text { RSS } \\
\text { Doubt }\end{array}$ \\
\hline Neuroticism & $.098 *$ & .049 & $.184 * *$ & $.100 *$ & $.291 * *$ & $.146^{* *}$ \\
\hline Gender & -.025 & -.030 & -.006 & -.037 & .062 & .012 \\
\hline Online Gambler & .049 & $.097 *$ & .080 & $.102 *$ & $.118 * *$ & $.091 *$ \\
\hline Chance Games & $-.123^{*}$ & .007 & -.042 & -.071 & .044 & -.078 \\
\hline Skill Games & -.012 & .015 & .013 & .024 & .047 & -.033 \\
\hline Age & .048 & -.090 & -.032 & -.030 & .034 & -.010 \\
\hline Annual Income & -.038 & -.032 & -.056 & -.033 & $-.096^{*}$ & -.047 \\
\hline Baseline RSS & $.608 * *$ & $.567 * *$ & $.590 * *$ & $.561 * *$ & $.459 * *$ & $.588 * *$ \\
\hline$R^{2}$ & .438 & .391 & .464 & .407 & .465 & .466 \\
\hline$F$ for $R^{2}$ & $32.47 * *$ & $26.71 * *$ & $36.01 * *$ & $28.57 * *$ & $36.24 * *$ & $36.32 * *$ \\
\hline \multicolumn{7}{|c|}{ STEP 2} \\
\hline Neuroticism & $.090 *$ & .037 & $.176^{* * *}$ & $.093 *$ & $.286 * *$ & $.137 * *$ \\
\hline Gender & -.025 & -.033 & -.007 & -.037 & .062 & .011 \\
\hline Online Gambler & .012 & .059 & .053 & .063 & $.092 *$ & .045 \\
\hline Chance Games & $-.129 *$ & -.001 & -.047 & -.078 & .038 & -.086 \\
\hline Skill Games & -.039 & -.014 & -.005 & -.002 & .030 & -.064 \\
\hline Age & .050 & -.089 & -.031 & -.031 & .035 & -.009 \\
\hline Annual Income & -.028 & -.021 & -.048 & -.023 & $-.089 *$ & -.036 \\
\hline Baseline RSS & $.569 * *$ & $.517 * *$ & $.576^{* *}$ & $.520 * *$ & $.450 * *$ & $.553 * *$ \\
\hline SOGS & $.141 * *$ & $.147 * *$ & $.087 *$ & $.136 * *$ & .078 & $.157 * *$ \\
\hline$R^{2}$ & .453 & .407 & .470 & .421 & .470 & .485 \\
\hline$\Delta R^{2}$ & .015 & .016 & .006 & .014 & .005 & .020 \\
\hline$F$ for $\Delta R^{2}$ & $9.19 * *$ & $8.85 * *$ & $3.88 *$ & $7.96 * *$ & 3.14 & $12.59 * *$ \\
\hline
\end{tabular}

Note: $* * p \leq 0.01 ; * p \leq 0.05 ; N=342$

Problem gambling severity in bold typeface for clarity. 